\title{
Binocular shape constancy from novel views: The role of a priori constraints
}

\author{
MOSES W. CHAN, ADAM K. STEVENSON, YUNFENG LI, and ZYGMUNT PIZLO \\ Purdue University, West Lafayette, Indiana
}

\begin{abstract}
We tested shape constancy from novel views in the case of binocular viewing, using a variety of stimuli, including polyhedra, polygonal lines, and points in 3-D. The results of the psychophysical experiments show that constraints such as planarity of surface contours and symmetry are critical for reliable shape constancy. These results are consistent with the results obtained in our previous psychophysical experiments on shape constancy from novel views in the presence of a kinetic depth effect (Pizlo \& Stevenson, 1999). On the basis of these results, we developed a new model of binocular shape reconstruction. The model is based on the assumption that binocular reconstruction is a difficult inverse problem, whose solution requires imposing a priori constraints on the family of possible interpretations. In the model, binocular disparity is used to correct monocularly reconstructed shape. The new model was tested on the same shapes as those used in the psychophysical experiments. The reconstructions produced by this model are substantially more reliable than the reconstructions produced by models that do not use constraints. Interestingly, monocular (but not binocular) reconstructions produced by this model correlate well with both monocular and binocular performance of human subjects. This fact suggests that binocular and monocular reconstructions of shapes in the human visual system involve similar mechanisms based on monocular shape constraints.
\end{abstract}

Shape constancy refers to the fact that the percept of the shape of a given object remains constant despite changes in the shape of the retinal image. The retinal image may change because of changes in the orientation of the object, relative to the observer. Shape is usually defined by angles and by ratios of distances. According to this definition, rigid motions and size scaling do not change shape. In other words, shape is invariant under similarity transformations. A perspective transformation between the 3-D scene and the 2-D retina is quite different from a similarity transformation (Pizlo, Rosenfeld, \& Weiss, 1997). First of all, it is a many-to-one mapping. As a result, the information about depth is lost, and the shape on the retina may change substantially when the orientation of the object, relative to the observer, changes. It follows that shape constancy is a difficult computational problem. In the case of familiar objects, shape constancy could, theoretically, be achieved by means of a simple template-matching

Partial reports of this study were presented at the annual meeting of the Association for Research in Vision and Ophthalmology (ARVO, 1999), of the Society for Mathematical Psychology (1999), of the Psychonomic Society (2000), and of the SPIE/IS\&T Electronic Imaging (2005). This research was done while M.W.C and A.K.S. were graduate students at Purdue University in the School of Electrical and Computer Engineering and in the Department of Psychological Sciences, respectively. This work was partially supported by the AT\&T Foundation, the Rockwell Foundation, and the National Science Foundation. The authors thank Allison Sekuler and the two anonymous reviewers for very useful comments and suggestions. Correspondence concerning this article should be addressed to Z. Pizlo, Department of Psychological Sciences, Purdue University, 703 Third Street, West Lafayette, IN 47907-2081 (e-mail: pizlo@psych.purdue.edu). mechanism, in which the current view is compared with all previously seen views of the object. In order to provide an adequate test between this and other mechanisms, one should use unfamiliar objects and test shape constancy from unfamiliar (novel) views.

Rock and DiVita (1987) performed the first systematic study of shape constancy from novel views, using 3-D objects. They used unstructured 3-D wire objects, which were viewed binocularly from a close viewing distance. Rock and DiVita reported a complete failure of shape constancy in the presence of a difference of only $45^{\circ}$ between the views of an object. The authors concluded that the shapes of 3-D objects are perceived (and recognized) by memorizing a large number of 2-D images taken from many different viewing directions - the template matching mentioned in the first paragraph. In a subsequent study, Rock, Wheeler, and Tudor (1989) provided additional evidence by showing that observers cannot imagine how a novel wire object looks from a new viewing direction. The results of these two studies strongly suggest that the perceptual representation of objects does not involve 3-D properties. Biederman and Gerhardstein (1993) reported a quite different result. They used line drawings of unfamiliar 3-D geometrical objects built from geons and demonstrated almost perfect shape constancy from novel views (geons are simple $3-D$ objects, such as a box, cone, cylinder, or pyramid; Biederman, 1987). This result is, actually, hardly surprising because, phenomenologically, it is easy to imagine how novel or familiar objects built from geons (and many other structured objects) look from new viewing directions. In other words, Biederman and Gerhardstein's study provided strong support for the claim 
that the perceptual representation of objects is 3-D and that this representation can be used to recognize objects. ${ }^{1}$ It follows that there are some fundamental differences in perceptual processing and representation between unstructured and structured objects. The most likely hypothesis is that 3-D perceptual representation is reliable in the case of structured objects, but not in the case of unstructured ones. In fact, in the case of structured objects, such as geons, a single image does give rise to a 3-D percept, whereas in the case of unstructured stimuli, such as wire objects, this is not the case.

As was indicated in the first paragraph, shape constancy is a difficult computational problem. Perspective projection from the 3-D scene to the 2-D retina removes information about depth. As a result, the inverse problem of reconstructing a 3-D shape from its 2-D image has infinitely many solutions. When two or more images are available, the 3-D scene may, in principle, be uniquely reconstructed, but the reconstruction tends to be unstable in the presence of visual noise (Pizlo, 2001; Poggio, Torre, \& Koch, 1985). The most effective way to overcome the computational difficulty of shape reconstruction is to impose a priori constraints on the family of possible 3-D interpretations. A number of constraints exist in the case of structured objects, but not in the case of unstructured ones.

A study reported by two of us (Pizlo \& Stevenson, 1999) tested the role of constraints in shape constancy from novel views, using both structured and unstructured 3-D objects. In that study, kinetic depth effect was the primary depth cue. In the first experiment, three types of stimuli were used. All the stimuli shared the same overall Euclidean structure characterized by 16 points, vertices of a symmetric polyhedron (see viper.psych.purdue.edu/shapedemo). In the first condition, the vertices were connected by the edges of the polyhedron. In the second condition, no edges were shown but only 16 points in $3-\mathrm{D}$. In the third condition, the points were connected in a random order by a polygonal line. So the only difference across the three conditions was whether and how the 16 vertices were connected. The first condition involved structured objects. The third condition involved unstructured objects, like the wire objects used by Rock and DiVita (1987). On each trial, two stimuli were presented successively, and the subject was asked to decide whether these stimuli were identical except for size and 3-D orientation. Since the magnitude of the 3-D differences among the 16 points that defined the stimuli in all three conditions and, thus, the magnitude of the 2-D differences among the images of the 16 points were the same across the three conditions, this study provided a fair test for the role of constraints in shape constancy of structured and unstructured objects. If the differences among the objects within conditions were not the same, the subjects' performance would confound the role of perceptual factors with the geometrical differences across conditions (Tjan \& Legge, 1998). Note that the second condition, which involved just 16 points, was actually expected to be more difficult than the other two, because the correspondence of points between the two objects to be compared was not provided to the subjects. The correspondence problem, however, was not an issue in the remaining two conditions. Pizlo and Stevenson showed that shape constancy was quite reliable in the case of structured objects (symmetric polyhedra) and was very unreliable otherwise (3-D polygonal lines and 16 points). These results demonstrated that shape constancy from novel views can be achieved in the case of structured objects that obey some regularity constraints (symmetry, planarity) but that it fails in the case of unstructured objects that do not obey any regularity constraints.

An anonymous reviewer has pointed out that these experiments do not necessarily establish that the shape of a polyhedron is perceived as the same when viewed from different viewing directions, but only that the subjects were able to identify the polyhedra accurately. In other words, perhaps the perceived shape changed somewhat from one view to another, but despite the changed percept of the shape of an object, the subject was able to identify that it was the same object. This is true, but high performance in this task implies that the subjects must have been able to use some information related to shape, such as higher order features characterized by affine or projective properties, that did not change between views (see, e.g., the study by Koenderink, van Doorn, \& Kappers, 1992). In the next two experiments, Pizlo and Stevenson used six additional types of stimuli, whose degrees of regularity varied systematically between that of a symmetric polyhedron and that of a polygonal line. The subjects' performance also varied systematically and was related to the degree of regularity of an object.

In the present study, we addressed a question as to whether shape constancy from novel views in the presence of a kinetic depth effect will generalize to binocular viewing. The three experiments reported below were analogous to the three experiments reported by Pizlo and Stevenson (1999). The results were quite similar to those reported by Pizlo and Stevenson, where the kinetic depth effect, rather than binocular disparity, was the cue to the three-dimensionality of the stimuli. Specifically, shape constancy was reliable when the stimuli are structured and was substantially less reliable otherwise. We conjecture that the visual system applies simplicity constraints but that these constraints are effective only in the case of stimuli that are structured. We then will present a hypothetical computational model of the underlying perceptual mechanisms.

\section{EXPERIMENT 1 Shape Constancy for Polyhedra, Vertices, and Polygonal Lines}

The first experiment tested the joint effect of several constraints. Experiments 2 and 3 tested the effect of individual constraints.

\section{Method}

Subjects. Four subjects were tested, including three of the authors. All of them had normal or corrected-to-normal vision. A.S., 
Z.P., and M.C. were experienced as subjects in psychophysical experiments, whereas A.M. was not. Z.P. and M.C. had prior experience as subjects in experiments with a stereoscopic display. A.S. and Z.P. received extensive practice before being tested. Specifically, they ran dozens of sessions with all types of stimuli before they ran the actual experiment. During this practice, they quickly reached the asymptotic performance, and the performance did not change over the course of a number of sessions. On the basis of this fact, the other 2 subjects received much less practice. This allowed for a reduction in the number of trials they had to run. The subject's head was supported by a chin-forehead rest, and the viewing distance was $50 \mathrm{~cm}$.

Stimuli. All the stimuli involved 16 points that were generated as vertices of a polyhedron. The positions of the vertices were generated randomly in 3-D, subject to the following constraints: The faces of the object were planar, the object had one plane of symmetry, the "front" part of the object was a box, which was smaller than the "back" part of the object (also a box), and the two boxes had a pair of faces that were coplanar.

Three types of stimuli were used. Stereoscopic images of one example of each stimulus are shown in Figure 1. The object depicted by the stimuli was represented by (1) the edges of the polyhedron (specifically, 16 planar contours represented the polyhedron), (2) the vertices of the polyhedron without any contours, or (3) a polygonal line connecting the vertices in a random order (see viper.psych .purdue.edu/shapedemo for more examples of stimuli for all the experiments reported in this article). Note that the stimuli in all three conditions had the same overall 3-D structure, involving 16 vertices of a polyhedron and differing only in the manner in which the vertices were connected (if at all).
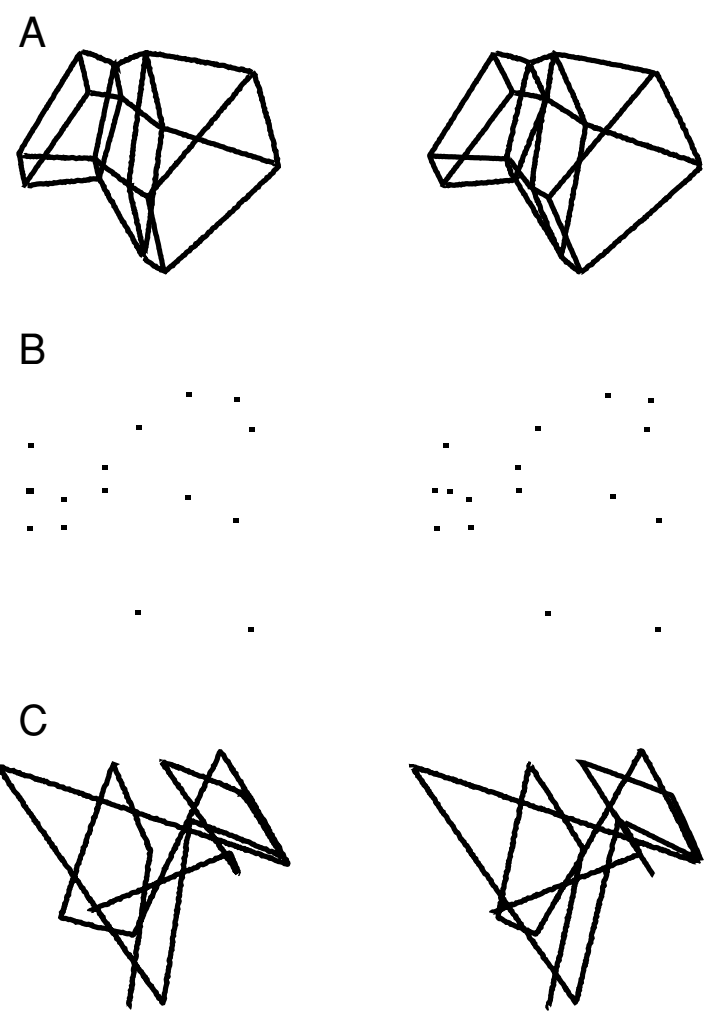

Figure 1. Stereoscopic images of one example for each type of stimuli used in Experiment 1 (for crossed viewing). (A) Polyhedron-planar, symmetric. (B) Vertices. (C) Polygonal line.
The three types of stimuli contained different constraints (if any). In Condition 1 (polyhedron), the contours were planar, the object had a plane of symmetry, and the images of the polyhedra were topologically stable (i.e., small changes in the 3-D orientation of the object did not lead to large topological changes in the image). Also, the shapes of the individual faces were not independent, because of the presence of topological information about which faces share edges and vertices. In other words, it is not possible to change the shape of one face of the polyhedron without changing the shapes of several other faces. In Condition 2 (vertices), only the symmetry constraint was present. In Condition 3 (polygonal line), no constraints were present.

Consider the role of the topological information that is present in the images of polyhedra in some more detail. It is interesting to observe that topological relations allow the application of some metric constraints. Take, for example, the constraint of minimum variance of angles (Marill, 1991). We can ask what shape of an $n$-gon (a polygon with $n$ vertices) minimizes the variance of angles. There is a unique solution to this minimization problem, and the solution corresponds to a regular $n$-gon, where all the angles are equal (the variance of the angles is zero). Such a polygon has a rotational symmetry of an order $n$; that is, the polygon, after being rotated around its center by $360 / n$ deg, is identical to itself. If, instead of a polygon, we take an open polygonal line with $n$ vertices (i.e., where the two endpoints of the line do not coincide), the task of minimizing the variance of the angles has infinitely many solutions. Namely, one can set the $(n-1)$ angles of the polygonal line to the same but arbitrary value $\alpha$, producing a minimal variance of angles equal to zero. Clearly, the constraint of minimum variance of angles is quite effective (a unique minimum) in the case of closed polygonal lines (polygons), but not in the case of open polygonal lines. The minimum variance of angles constraint leads to a symmetric polygon only in the absence of additional constraints. However, when there are additional constraints provided by the retinal image (i.e., the reconstructed polygon in 3-D space is expected to be perspectively equivalent to the polygon on the retina), the minimum variance of angles may lead to a nonsymmetric polygon. In this case, the resulting polygon will be as close to a symmetric one as the retinal image allows. Additional discussion of the minimum variance of angles constraint will be presented in the Computational Model section.

The stimuli were displayed by means of a stereoscopic display. The left and right images were computed using the same method as that described in Chan, Pizlo, and Chelberg (1999). The simulated viewing distance was $50 \mathrm{~cm}$, and the simulated interocular distance was $6.5 \mathrm{~cm}$. The switching rate of the display was $144 / \mathrm{sec}$. The luminance of the object through the active LCD glasses was $1.5 \mathrm{~cd} / \mathrm{m}^{2}$, and the luminance of the background was less than $0.01 \mathrm{~cd} / \mathrm{m}^{2}$. The experiment was performed in a dark room. Under these viewing conditions, the stimuli were clearly visible.

Each trial involved two objects whose shapes were either the same or different. First, consider the trial with the same shapes. At the beginning of the trial, an object was displayed at a random 3-D orientation relative to the reference frame. After $1 \mathrm{sec}$, the object disappeared. Then the object was rotated by $90^{\circ}$ around the vertical axis and was displayed again for $1 \mathrm{sec}$ (the interstimulus interval was equal to $1 \mathrm{sec}$ ). As a result, the views (images) in the two presentations were different, regardless of whether the objects themselves were or were not the same. A small random rotation (up to $\pm 20^{\circ}$ ) around the other two axes, size scaling (up to $\pm 50 \%$ of the object size) and horizontal/vertical translations (up to $\pm 10 \%$ of the image size) were added in order to minimize 2-D artifactual cues. In a trial with different shapes, the second presentation involved a new, randomly generated object. This different object was rotated relative to the frame of reference, translated, and scaled identically as in the trial with same shapes. In Condition 3, where a polygonal line connected the vertices in a random order, the order was the same in both presentations of a given trial, but it changed between trials. 
Procedure. A signal detection experiment with confidence rating was used (five levels of confidence were used). The discriminability $d^{\prime}$ was estimated by $d$, which is the distance between the ROC and the origin times $\sqrt{2}$ (Macmillan \& Creelman, 2005, pp. 59-64). Each session consisted of 200 same and 200 different trials, presented in a random order (each session started with 40 practice trials). The subject's task was to discriminate whether the two objects had the same or different shapes (i.e., whether the 3-D stimuli were identical up to rotation, translation, and size scaling). After each trial, the subject received feedback about the accuracy of the response. The subjects were asked to distribute their responses as uniformly as possible among the five confidence levels. If most responses involve only two or three confidence levels, the discriminability $d^{\prime}$ and its standard error cannot be reliably estimated (Macmillan \& Creelman, 2005). The stimulus type was constant within a given session, and the order of the sessions was random and different for every subject. For example, 1 subject started with the polyhedron condition and ended with the vertices condition. A 2nd subject ran these sessions in the opposite order. And so on. Even though we were rather confident that the subjects had reached an asymptotic performance before the experiment started (this was directly verified in the case of A.S. and Z.P.), we wanted to avoid any order effects, in which case the differences among conditions might have been attributable to the order of the conditions used.

\section{Results and Discussion}

In all three conditions, the subjects reported that they clearly saw the stimuli as 3-D. This means that the stereoscopic display, which simulated binocular viewing of 3-D objects, provided effective depth cues in all three conditions. However, this clear perception of three-dimensionality did not lead to equally good performance in the shape constancy task. The results are shown in Figure 2. The ordinate shows $d^{\prime}$. A higher $d^{\prime}$ represents better (more accurate) performance. The pattern of results is similar in all the subjects, although 1 subject (A.M.) performed relatively poorly. (A.M. was inexperienced as a subject, and furthermore, he received the smallest amount of practice. Small amount of practice is likely to lead to instability of the response criteria, which in turn leads to an underestimation of $d^{\prime}$.) We used an ANOVA to evaluate the statistical significance of the results. The three conditions led to different performance $(p<.01)$. Specifically, the polyhedron condition led to much better performance than did the other two conditions $(p<.02)$, and the performance in these two conditions was close to chance level $\left(d^{\prime}=\right.$ 0.43 in the case of the vertices and 0.15 in the case of the polygonal line; $p>.05$ ). These results clearly show that the shapes of objects can be reliably recognized when the objects contain a number of constraints and shapes may not be reliably recognized when all constraints are absent. These results are quite similar to those reported by Pizlo and Stevenson (1999), where the kinetic depth effect, rather than binocular disparity, was used as a depth cue.

It is worth pointing out that performance in the vertices condition was somewhat better than that in the polygonal line condition (although this difference was not significant statistically). Geometrically, the vertices condition should be most difficult, because the correspondence of the 16 points across the two stimuli was not provided to the subjects. In particular, even if the two presentations involved the same 3-D stimulus (up to size scaling and 3 -D rotation), the subjects might have responded different

\section{Experiment 1}
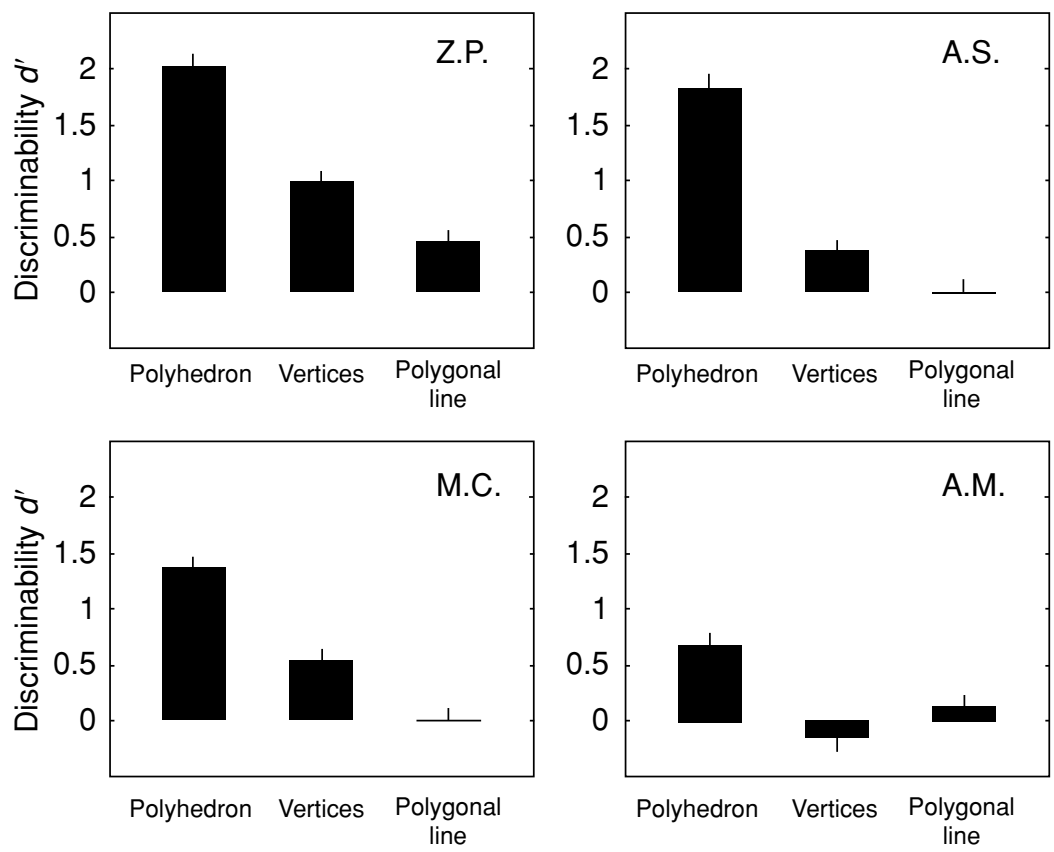

Figure 2. Results of Experiment 1. The ordinate shows $d^{\prime}$. The error bars show plus or minus the standard deviation of the mean. 
if they failed to figure out which point in one stimulus went with which in the other. In contrast, the polygonal line condition, which involved the same set of 16 points, provided information about the correspondence of the points across the two stimuli in each trial, because the order in which the points were connected by the line, was the same. Despite the fact that correspondence was less of a problem in the polygonal line than in the vertices condition, performance in this condition was the worst. Apparently, the 3-D mirror symmetry that was present in the vertices, but not in the polygonal line, condition was perceptually more important than explicitly given correspondence in the latter.

Our results in the polyhedron condition are quite similar to those in Biederman and Gerhardstein (1993), in that shape constancy was reliably achieved. It seems, therefore, that shape constancy can be observed not only for objects composed of geons, but also for other structured objects. In particular, in our experiment, all the stimuli in the polyhedron condition had the same topological structure with the same number of edges, vertices, and contours. Next, our results in the polygonal line condition are quite similar to those in Rock and DiVita (1987), in that shape constancy completely failed. But unlike in those previous studies, in which the stimuli were very different (geons vs. wires), our stimuli were characterized by the same (or at least a quite similar) degree of geometrical differences within each condition (see the Method section).

One of the anonymous reviewers indicated that the polyhedron and the polygonal line stimuli have different complexity and, therefore, one should be cautious in drawing conclusions from the result that performance in the former is much higher than that in the latter. Specifically, the polyhedron contained 28 edges, whereas the polygonal line contained only 15 . Next, the polyhedron contained vertices from which 3 or 4 edges emanated, whereas the polygonal line contained vertices from which, at most, 2 edges emanated. The fact that this difference is not critical will be shown in Experiment 2, in which a nonplanar, asymmetric polyhedron was used. This polyhedron was identical to the polyhedron in Experiment 1, in that it contained 28 edges and had vertices from which 3 or 4 edges emanated. However, performance in this condition was closer to that in the polygonal line condition than to that in the polyhedron condition in Experiment 1. Next, the reviewer indicated that in the case of the polyhedron in Experiment 1, there is only one way to connect the vertices so that a symmetric polyhedron with planar contours is formed, whereas in the case of the polygonal line, there are 16 ! ways of connecting the vertices. The fact that this difference is not likely to be critical will be shown in Experiment 3 , where 10 points, forming three planar quadrilaterals, were used. This stimulus (three quadrilaterals) was identical to that in the polyhedron condition in Experiment 1 , in that there was only one way to connect the 10 points to form the stimulus (here, planar quadrilaterals). However, performance in this condition was clearly lower than that in the polyhedron condition in Experiment 1.

To summarize, our psychophysical results suggest that the difference in the degree of shape constancy between structured and unstructured objects is critically related to the availability of effective constraints. Our simulation studies reported in the second part of the article suggest, in turn, that when effective shape constraints can be applied, the 3-D perceptual representation of shape is reliable and that the perceptual representation may be quite unreliable if constraints are not applied. These results are consistent with the observation stated in the beginning of this article, that it is easy to imagine how a structured object looks from another view but that this is almost impossible in the case of an unstructured object.

The next step was to test the effects of the individual constraints, in order to determine whether all of them are equally important. These tests were performed in the next two experiments. These experiments used essentially the same method as that in Experiment 1.

\section{EXPERIMENT 2}

\section{The Effect of Planarity and Symmetry}

\section{Method}

Subjects. Two of the authors (A.S. and Z.P.) and two naive subjects were tested.

Stimuli. Three types of stimuli were used (see Figure 3). (1) The first type was a partially nonplanar, symmetric polyhedron. Obviously, a polyhedron has, by definition, planar faces, and contours representing these faces are planar, too. However, in the case of the stimuli in this condition, some of the contours that were planar in Condition 1 in Experiment 1 were no longer planar here. The
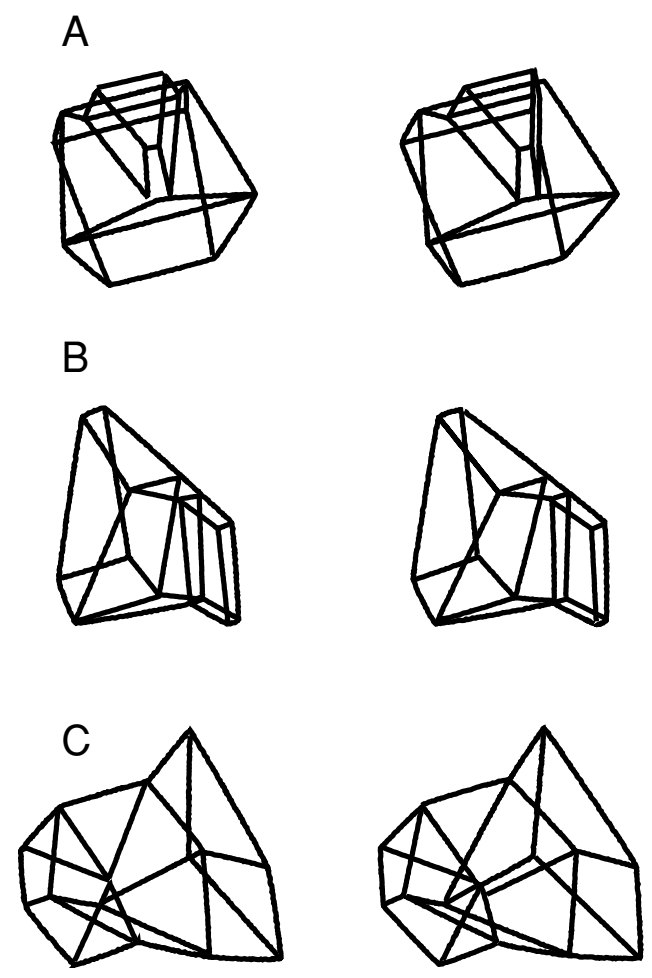

Figure 3. Stereoscopic images of one example for each type of stimuli used in Experiment 2. (A) Partially nonplanar, symmetric. (B) Planar, asymmetric. (C) Nonplanar, asymmetric. 
planarity constraint of the contours of faces was removed, but the polyhedron still had a plane of symmetry. It is easy to show that if a quadruple of points have a plane of symmetry, so that none of the points is on this plane, this quadruple is coplanar. Thus, even though the planarity constraint was not explicitly used in generating the stimuli in this condition, the symmetry constraint implied planarity of some of the contours. (2) The second type was a planar, asymmetric polyhedron. This stimulus was obtained by using only one half of the polyhedron from Condition 1 in the first experiment (more specifically, one of the two symmetric parts). Note that this stimulus also had 16 vertices and 16 contours, like all the other polyhedra in Experiments 1 and 2, except that now, several vertices happened to be collinear (see Figure 3B). (3) The third type was a nonplanar, asymmetric polyhedron. The contours of this polyhedron were nonplanar, and the polyhedron was asymmetric.

In the partially nonplanar, symmetric condition, the only constraint that was removed, as compared with the polyhedron condition, was that not all the contours were planar. In the planar asymmetric condition, the symmetry constraint was removed. In the nonplanar, asymmetric condition, both planarity and symmetry constraints were removed. The order of individual experimental conditions (sessions) was random and different for the 4 subjects. Since the 2 naive subjects did not participate in Experiment 1, they were also tested in the polyhedron condition from that experiment.

\section{Results and Discussion}

Figure 4 shows the results. In the case of the authors, on this graph, we plotted a bar representing their performance in the polyhedron condition in Experiment 1. It can be seen that performance was not identical across the four conditions $(p<.002)$. Removing the planarity constraint in some of the contours harmed the performance in the case of all the subjects, although this effect was not significant $(p>.1)$. Removing the symmetry constraint led to a similar deterioration of performance $(p>.05)$. Removing both the planarity and the symmetry constraints led to a substantial drop of performance for all the subjects. Specifically, performance in the nonplanar asymmetric condition was significantly lower than that in the other three conditions $(p<.005)$. These results indicate that human shape perception involves constraints of planarity of contours and, also, symmetry of the object.

Liu and Kersten (2003), using simple polygonal line objects, also demonstrated an advantage for symmetric objects, although their task was different from ours. In the symmetric condition, the reference and the test objects were symmetric, whereas in the asymmetric condition, the reference objects were symmetric and the test objects were asymmetric. Liu and Kersten concluded that the advantage for symmetric objects is related to the economy of perceptual representation. Interestingly, the symmetry of a 3-D object may improve economy of perceptual representation not only when the representation is 3-D, but also when it involves a set of 2-D images (views) of a 3-D object. Vetter, Poggio, and Bülthoff (1994) showed that

\section{Experiment 2}
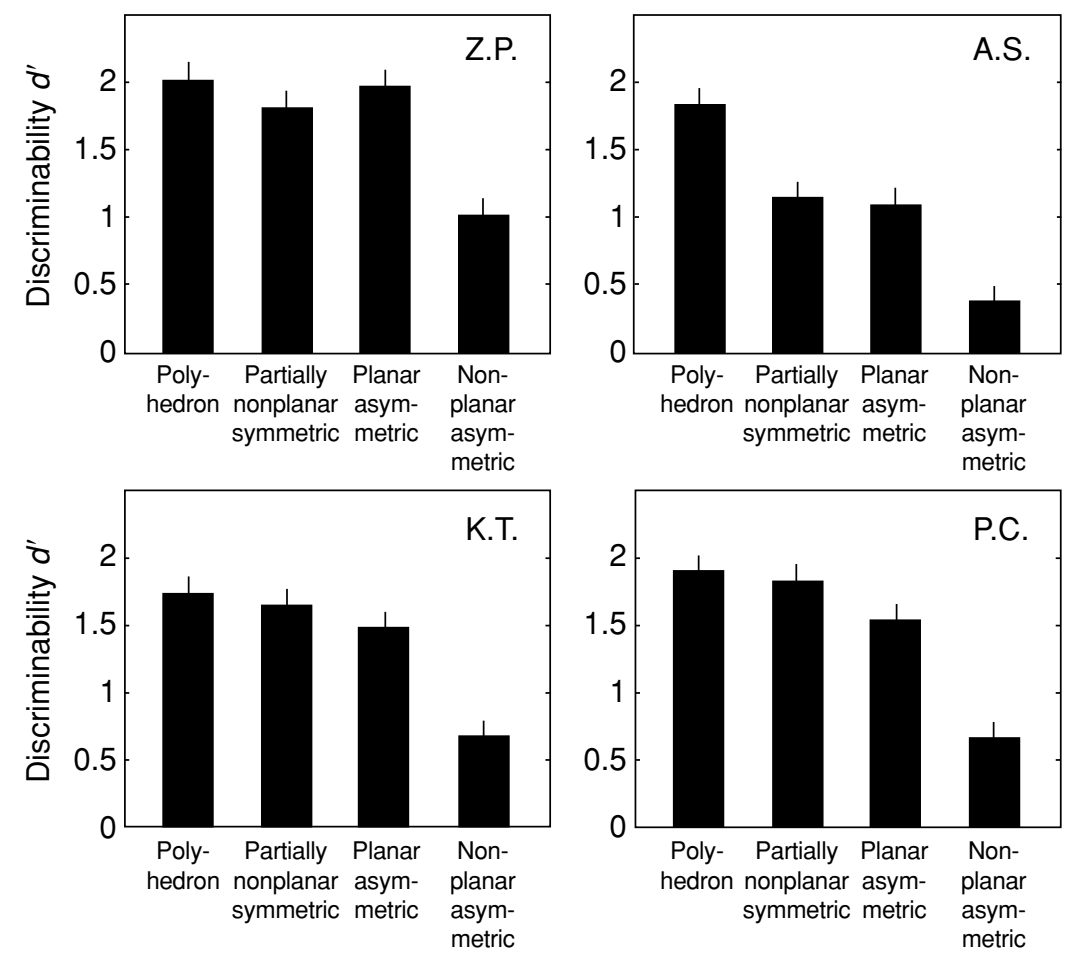

Figure 4. Results of Experiment 2. The results for the polyhedron condition in Experiment 1 are also shown for comparison. The ordinate shows $d^{\prime}$. The error bars show plus or minus the standard deviation of the mean. 
given some images of a symmetric object, new images of the object can be computed without knowledge of the 3-D object itself.

The next experiment was designed to test the effect of constraints provided by topological information about which contours share edges and vertices. As is indicated in the Method section of Experiment 1, the shapes of planar contours in the polyhedron condition were not independent. As a result, the shapes of contours provide redundant information about the shape of a polyhedron. Perhaps more important, topological relations make it possible to apply constraints, such as the minimum variance of angles described earlier in this article. Marill (1991) and then Leclerc and Fischler (1992) and Sinha and Adelson (1992) have argued that this constraint leads to perceptually plausible reconstructions of polyhedra from a single image. To partially remove topological information from the stimulus, we presented only three faces of the polyhedron (three quadrilaterals) in such a way that each of two pairs of the faces shared only one vertex (see Figure 5A). As a result, the shapes of the three contours were independent. If joint constraining among the shapes of contours is critical to binocular shape perception, this stimulus should lead to deterioration of performance, in comparison with the polyhedron stimulus.

\section{EXPERIMENT 3 The Role of Topological Relations}

\begin{abstract}
Method
Subjects. The 4 subjects who had participated in Experiment 2 were tested.

Stimuli. Three types of stimuli were used (see Figure 5). (1) The first consisted of the three faces (quadrilaterals) of the polyhedron. Each of two pairs of the quadrilaterals shared one vertex. Thus, the topological relations were very weak. (2) The second consisted of three triangles. Each of the triangles was one part of each quadrilateral that was used in Condition 1. Here, the topological relations were equally weak as those in the previous condition. Using triangles, rather than quadrilaterals, allowed additional testing of the planarity constraint. Although triangles are planar, the planarity constraint is weaker, in comparison with quadrilaterals, since any three, but not any four, points are coplanar. (3) The third type was a polygonal line connecting the seven vertices that were used in the previous condition. Specifically, this stimulus was obtained by removing one side from each of the three triangles. Again, the topological relations were very weak. The polygonal line in this experiment was different from the polygonal line in Experiment 1 in that it had fewer vertices ( 7 vs. 16). Furthermore, the polygonal line in this experiment, led to many fewer intersections in the image, and thus, the image was topologically more stable. The order of conditions was random and different for the 4 subjects.
\end{abstract}

\section{Results and Discussion}

The results are shown in Figure 6 (again, we show the performance in the polyhedron condition of Experiment 1). It can be seen that removing the topological relations harmed the performance substantially: The differences across the four conditions were statistically significant $(p<.01)$. Performance in the polyhedron condition was higher than that in each of the other three conditions $(p<.025)$. This drop in performance is con-
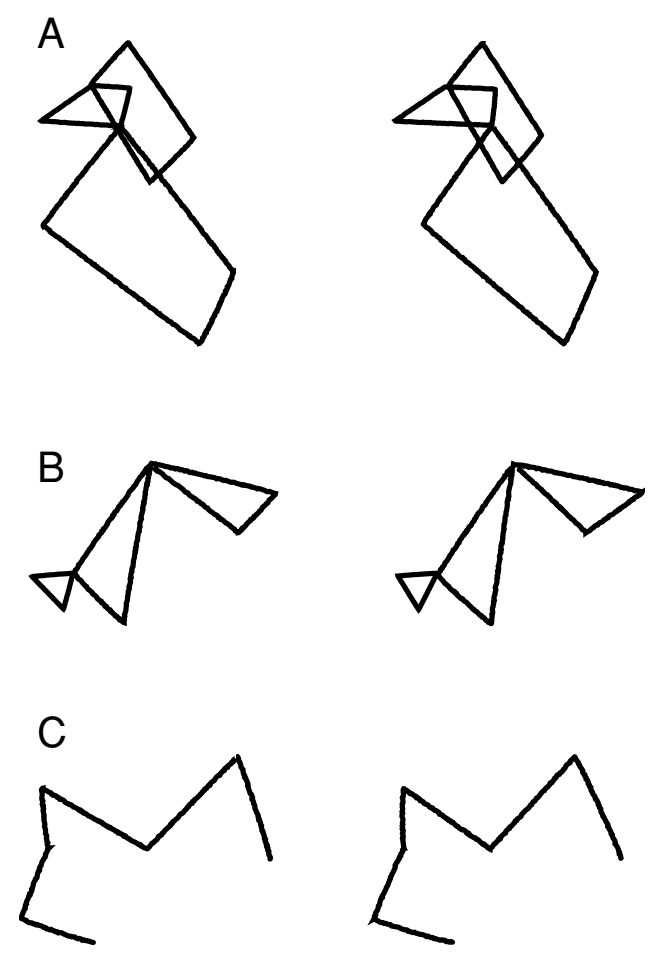

Figure 5. Stereoscopic images of one example for each type of stimuli used in Experiment 3. (A) Three quadrilaterals. (B) Three triangles. (C) Polygonal line.

sistent with our prediction about the importance of constraining among the shapes of faces. Performance in the triangles condition was significantly lower than that in the quadrilaterals condition $(p<.05)$, although this difference was rather small. Finally, performance in the polygonal line condition was about the same as that in the triangles and quadrilaterals conditions $(p>.25)$. Finally, note that performance in the polygonal line condition in this experiment was somewhat higher than that in Experiment 1.

To summarize, constraints, such as planarity of surface contours, nonindependence of the shapes of contours due to topological relations among the contours, and symmetry, are critical in binocular shape constancy. Interestingly, these constraints closely resemble the constraints that were previously identified, using various psychophysical methods and stimuli, in perception of shape from (1) a single image (Attneave \& Frost, 1969; Hochberg \& Brooks, 1960; Hochberg \& McAlister, 1953; Perkins, 1972, 1982; Sinha \& Adelson, 1992), (2) motion (Pizlo \& Stevenson, 1999), and (3) binocular (stereoscopic) matching (Mitchison, 1988). The fact that shape perception in human observers involves the same set of constraints in the cases of perception from a single image, from two images (binocular), and from multiple images (motion) provides strong support for the claim that all these cases involve similar perceptual mechanisms. In the next section, we will present a new model for binocular shape constancy. Because the existing experimental results are not sufficient to pro- 


\section{Experiment 3}
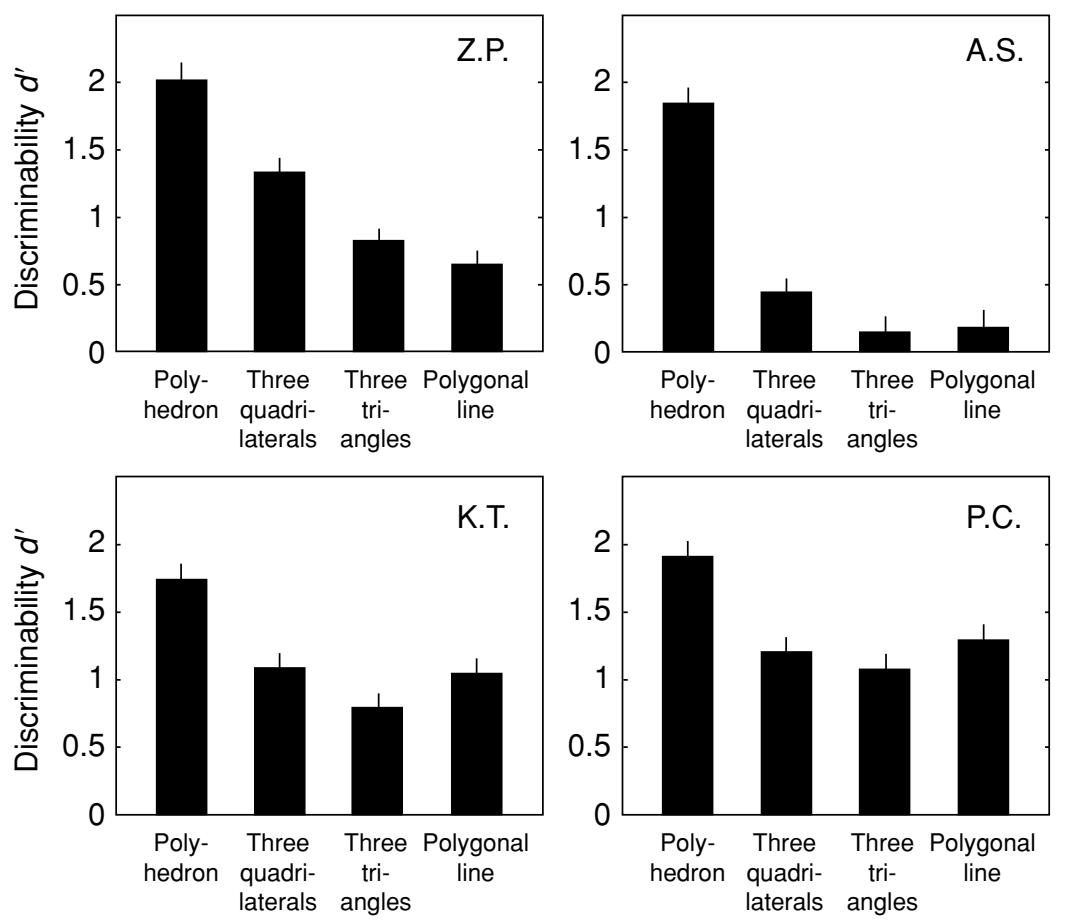

Figure 6. Results of Experiment 3. The results for the polyhedron condition in Experiment 1 are also shown for comparison. The ordinate shows $d^{\prime}$. The error bars show plus or minus the standard deviation of the mean.

pose a complete theory of shape constancy, we concentrated on the case of reconstructing shapes. Specifically, we posed two related tasks: (1) formulate a model that is computationally effective (i.e., that can reliably reconstruct 3-D shapes) and (2) test this model with respect to psychological plausibility. A rationale for using this twostep approach will be given below.

As was indicated in the introductory section, the task of reconstructing a 3-D shape in general, and from binocular disparity in particular, is computationally difficult. The main problem is related to the fact that the information about depth is largely lost from retinal images and that cues to depth, such as binocular disparity, motion, shading, and texture, are quite unreliable. As a result, the reconstruction of depth relations, such as the length of a single 3-D line segment, is very imprecise and may be quite inaccurate (e.g., McKee, Levi, \& Bowne, 1990; Norman, Todd, Perotti, \& Tittle, 1996). Specifically, the perceived 3-D length varies from trial to trial substantially (Weber fractions are as large as $30 \%$ ), and it is strongly affected by the viewing conditions (the systematic errors are as large as $50 \%$ for a $150-\mathrm{cm}$ viewing distance). It is not difficult to emulate, with a computational model, human performance in the reconstruction of 3-D distances, because the task is theoretically simple and human performance is quite poor. What is very difficult, however, is to emulate human performance in the reconstruction of the shapes of 3-D objects. Chan et al. (1999) implemented a model of reconstructing 3-D distances and ratios of distances, using binocular disparity. Performance of this model was equally good or better than that of other models that do not use shape constraints. Also, the performance of this model was equally poor as that of human subjects in a 3-D distance task, but at the same time, it was an order of magnitude worse than that of human subjects in the reconstruction of the shapes of 3-D structured objects. These results strongly suggest that human perception of $3-\mathrm{D}$ shapes is not derived from perception of 3-D distances. Instead, perception of 3-D shapes involves a combination of simplicity constraints and binocular disparity (Chan et al., 1999).

On the basis of these facts, we decided to formulate a computational model for binocular shape reconstruction that involves constraints and (1) whose performance in reconstructing 3-D shapes is substantially better than that of Chan et al.'s (1999) model and (2) that is psychologically plausible. Task 1 is a necessary condition for Task 2 . In this article, we will describe a model that satisfies Task 1 , and we will outline directions for future research that is likely to lead to accomplishing Task 2.

\section{COMPUTATIONAL MODEL OF BINOCULAR SHAPE RECONSTRUCTION}

There are two general methods of incorporating a priori constraints into models of perceptual reconstruction: 
probabilistic, involving the likelihood (Bayes's) principle, and deterministic, involving the simplicity principle (e.g., Kersten, Mamassian, \& Yuille, 2004; Pizlo, 2001; Pomerantz \& Kubovy, 1986). Each of these methods can be reformulated in the language of the other, and therefore, the choice between these two methods depends largely on the assumptions about nature of the constraints. Because the constraints that we identified can be treated as simplicity constraints, our model is based on deterministic approach.

Next, note that the constraints that we identified allow reconstructing a 3-D shape on the basis of either one or more than one retinal image. Because reconstructing a 3-D space directly from binocular disparity is a difficult inverse problem, which is likely to lead to unstable results (see Chan et al., 1999), our model begins 3-D reconstruction by applying constraints to a 2-D image. The fact that monocular reconstruction of 3-D shapes with the use of simplicity constraints may lead to reliable results has been demonstrated by Marill (1991) and by Leclerc and Fischler (1992). After the monocular 3-D reconstruction is completed, our model uses binocular disparity to correct the monocularly reconstructed shape. ${ }^{2}$ Our conjecture that the role of binocular disparity may be limited to that of correcting a monocularly reconstructed 3-D shape receives support from recent psychophysical experiments performed by two of us (Pizlo, Li, \& Francis, 2005). These experiments showed that in the case of structured stimuli such as polyhedra, the binocular percept is dominated by 3-D monocular cues, such as information about which parts represent a single object, as well as constraints that lead to 3-D reconstruction from a single 2-D image.

It is assumed in our model that the feature (point) correspondences are known to the observer's visual system. In other words, our model does not solve the binocular correspondence problem but, instead, assumes that this problem has already been solved.

\section{Applying Constraints to a 2-D Image}

The first computational stage in our model is a reconstruction of a 3-D shape on the basis of one retinal image, $I$ (the model performs two such reconstructions, one for each image). Henceforth, this shape will be called a shape hypothesis, $H$. A single 2-D image determines infinitely many possible 3-D shapes. In order to choose one, several constraints are used. Our algorithm for performing shape reconstruction from one image is based on Leclerc and Fischler's (1992) algorithm. In their algorithm, the 3-D shape of a polyhedron was obtained from a single orthographic image by taking the individual points of the 2-D image and "moving" them along the lines orthogonal to the image plane. This was done by first assigning the same $z$-coordinate to every image point. Then, the $z$-coordinates of the points were changed independently, while keeping the $x$ - and $y$-coordinates unchanged. As a result, any 3-D object produced by this algorithm was consistent with the given image under orthographic projection. From the infinite set of such objects, the algorithm chose the one that minimized some complexity measure represented by a cost function. This function had two components: the magnitude of the overall departure of reconstructed faces from planarity and the variance of all the interior angles. As was indicated above, the minimum of variance of angles can be interpreted as a weak measure of symmetry. For example, when this constraint is applied to an image of a cube, it will produce a cube. When a perfect cube is not perspectively consistent with the retinal image, the minimum variance of angles constraint will produce an object that is approximately a cube.

In our implementation, we used a perspective, rather than orthographic, projection, and our cost function included also the magnitude of the departure of the reconstructed object from a mirror symmetry. For the shape hypothesis computed from the left image, the cost function has the following form (the shape hypothesis computed from the right image is analogous):

$$
\begin{aligned}
E_{\text {mono }}(H) & = \\
\gamma & {\left[S D A^{2}(H)+D S(H)\right]+(1-\gamma) D P(H), }
\end{aligned}
$$

where $S D A^{2}(H)$ is the variance of all interior angles, $D P(H)$ is a measure of departure from planarity for all the faces of $H, D S(H)$ is a measure of departure from mirror symmetry of $H$, and $\gamma$ is a scalar that ranges from 0 to 1 .

It is assumed in the model that the plane of symmetry is known. More exactly, it is assumed that it is known which angles are the symmetric ones. The departure from mirror symmetry is computed as the sum of the squared differences between the corresponding angles over all the pairs of angles in the polyhedron. The departure from planarity was measured by using a property of polygons according to which the sum of all interior angles in an $n$-gon is equal to $(n-2) * 180^{\circ}$. When a convex polygon is not planar, the sum of interior angles is smaller. Thus, the following expression is a measure of the departure from planarity of a convex $n$-gon (Leclerc \& Fischler, 1992):

$$
D P=\left[(n-2) \cdot 180-\sum_{j} \alpha_{j}\right]^{2} \text {. }
$$

In Equation 1, the term $D P(H)$ is the sum of the departure from planarity over all contours. Note that all three constraints are commensurate - that is, all use $(\mathrm{deg})^{2}$ as a unit. The role of $D S(H)$ and $D P(H)$ in Equation 1 is selfexplanatory. The role of the variance of angles is less obvious. We indicated above that this term can be interpreted as a weak measure of the symmetry of a polyhedron. This term was used for the first time by Marill (1991). He showed that minimum variance of angles by itself led to good reconstructions. Leclerc and Fischler (1992) have pointed out that this term is important in producing a volume of the reconstructed object. They added the planarity constraint to improve the reconstructions. The minimum variance of angles and the symmetry constraints are given equal weights in the cost function (Equation 1). We tried other weight combina- 
tions for these two constraints and found that they had only a small effect on the quality of the reconstruction.

Following Leclerc and Fischler (1992), we apply a continuation method (Leclerc, 1989; Witkin, Terzopoulos, \& Kaas, 1987) to minimize $E_{\text {mono }}(H)$. This method involves a sequence of descent steps applied to $E_{\text {mono }}(H)$, in which $\gamma$ is decreased in each step. The algorithm starts with $\gamma \approx 1$. This means that the first step is strongly influenced by $S D A^{2}(H)$ and $D S(H)$ terms, which give the initial shape (volume) of $H$. As $\gamma$ decreases, $D P(H)$ becomes the dominant factor, which enforces planarity of the faces. It is not desirable to emphasize planarity in the beginning of the reconstruction process. Note that the image itself is planar and, thus, represents a local minimum of the cost function (Equation 1). To "escape" from this minimum, planarity constraint is assigned a small weight in the beginning of the reconstruction.

\section{Correcting the Shape Hypothesis}

At this stage, $H$ is still an initial "guess" of the 3-D shape of the object. The model has to incorporate information from the second image, $I^{\prime}$ in order to obtain a more accurate reconstruction. Thus, a measure of similarity between $H$ and $I^{\prime}$ is needed, where $H$ is the shape hypothesis produced from the left retinal image, $I$, and $I^{\prime}$ is the right retinal image (and vice versa). Here, the similarity $\operatorname{SHI}\left(I^{\prime}, H\right)$ is the sum of squared differences between the angles of $I^{\prime}$ and the corresponding angles of $H$ projected on the right retina. In order to project $H$ on the right retina, it is necessary to know the position and orientation of one eye, relative to the other. More exactly, one needs to know the three angles characterizing the 3-D orientation and two out of the three distances characterizing the 3-D position. The knowledge of the interocular distance is not needed if the object's shape, but not size, is to be reconstructed (Pizlo, Rosenfeld, \& Weiss, 1995). In this article, we assume that these five parameters are computed from the retinal information (the interocular distance is set to some arbitrary value-e.g., 1.0). The fact that these five parameters can be computed from the visual information itself (more exactly, from at least eight points in 3-D) was demonstrated by Longuet-Higgins (1981). Our algorithm uses a modification of Longuet-Higgins's algorithm in which fewer 3-D points are needed (Chan et al., 1999).

Binocular shape correction is performed in our model by changing only the global features of the shape hypothesis. This ensures that the second image will not lead to instability of the reconstruction. Specifically, we correct $H$ by applying 3-D projective transformations to the two parts (boxes) of the object independently. The set of all 3 -D projectivities is characterized by 15 free parameters (Faugeras, 1993). We use a subset of all 3-D projectivities, which guarantees that the object after a transformation produces the same image on the left retina as the object before the transformation. In other words, the object points must stay on their projecting lines. This subset of 3-D projectivities is characterized by 4 parameters (see Chan, 1999, for mathematical details). The algorithm finds the values of these parameters, which minimize the following cost function:

$$
E_{\text {bino }}(H)=S H I\left(I^{\prime}, H\right)+\lambda\left[S D A^{2}(H)+D S(H)\right] .
$$

Our simulations were performed with $\lambda=1$, but the performance of the algorithm did not change much for other values in the range between 0.1 and 3.0. Two minimization problems were solved: one for each image. The final shape was selected from the two shape hypotheses $H$ and $H^{\prime}$ as the one that has a smaller value of $E_{\text {bino }}$. Note that there is no planarity constraint in this cost function. This constraint was not used, because the family of projective transformations, which is used in binocular correction, preserves the planarity of contours produced in monocular reconstruction. However, if the monocular reconstruction produced nonplanar contours, the contour remained nonplanar after binocular correction.

\section{SIMULATION EXPERIMENTS}

We tested our new model by using polyhedral stimuli from our Experiment 1 (the first three tests). The last test used also the polyhedra from Experiment 2. The objects were placed at the (simulated) distances that corresponded to vergence angles of $8^{\circ}, 4^{\circ}$, and $2^{\circ}$.

Noise that is added by the visual system to a retinal image was an important factor in the simulations. We wanted to use noise whose statistical properties are similar to those characterizing the noise in the human visual system. It is commonly assumed that the noise in the visual system can be approximated by a Gaussian probability density function with zero mean. The main question was about the standard deviation of this distribution. Because the density of receptors on the retina is not constant, therefore, it is reasonable to assume that the magnitude of uncertainty about spatial position (standard deviation of noise) varies across the retina. More exactly, this uncertainty is approximately proportional to eccentricity (Anstis, 1974; Pizlo, Rosenfeld, \& Epelboim, 1995; Rawlings \& Shipley, 1969). But what is the coefficient of proportionality? This coefficient depends on the stimulus, the viewing conditions, and the subject's task, indicating the existence of more than one source of noise. Each source of visual noise is associated with a corresponding visual acuity. The acuity that is often discussed in the context of binocular vision is called stereoacuity. Stereoacuity belongs to the class of hyperacuities (e.g., Regan, 2000), which involve judgments of relative position, such as whether the target is in front of or behind the reference. These judgments involve spatial precision of several seconds of arc, which is an order of magnitude less than the distance between receptors. Hyperacuities, however, are not directly relevant to our model, where the problem is to reconstruct a shape. One way to characterize shape is to use ratios of distances among all the pairs of points of an object or a figure, especially large distances, which more closely characterize global aspects of the object's or figure's shape. In a task of judging the length of a long line segment on the retina (longer than the 
eccentricity of the endpoints), the difference threshold is proportional to the eccentricity of the endpoints, and the coefficient of proportionality is equal to $1 \%-3 \%$ (Levi \& Klein, 1990; for a review, see Pizlo, Rosenfeld, \& Epelboim, 1995). There are no data characterizing the effect of eccentricity on binocular difference threshold in judging distances in depth. Existing results for stimuli around the fixation point show that the binocular difference threshold is larger by a factor of two than the monocular one (McKee et al., 1990). To approximate visual noise in our simulations, we used uncorrelated Gaussian noise with zero mean and standard deviations of $1 \%, 2 \%, 3 \%$, and $5 \%$ of the diameter of the object's image (this corresponds to $2 \%$, $4 \%, 6 \%$, and $10 \%$ of the maximal eccentricity of the retinal stimulus). This noise was imposed on each image coordinate. One percent of noise for an individual point leads to $1.4 \%$ of uncorrelated noise for the distance between two points when expressed as a function of the diameter of the stimulus and about $3 \%$ when expressed as a function of eccentricity. This level of noise corresponds to the monocular noise in the human visual system and is a lower bound of the binocular noise, when the observer makes global judgments about distances.

For each condition, we generated randomly 100 objects. The accuracy of shape reconstruction was evaluated by computing ratios of pairwise distances (recall that shape is characterized by ratios of distances, not by the distances themselves). Because an object has 16 vertices $U_{i}(i=1$, $\ldots, 16)$, there are 120 pairwise distances between points $U_{i}$ and $U_{j}$. The longest distance was used as the length of the object and was denoted $L$. For the remaining 119 distances, we computed ratios

$$
R_{i j}=\left\|U_{i}, U_{j}\right\| / L,
$$

where $\left\|U_{i}, U_{j}\right\|$ denotes the Euclidean distance between points $U_{i}$ and $U_{j}$.

Let $R_{i j}^{j}$ be computed from the original object and $R_{i j}^{\prime}$ from the reconstructed object. If the shape of an object is reconstructed accurately, then $R_{i j}=R_{i j}^{\prime}$ for all pairs of points. In the presence of noise, these ratios are never equal exactly. But they are equal approximately. To evaluate the quality of the reconstruction, we computed a ratio of these ratios (henceforth, called normalized ratio $r$ ):

$$
r_{k}=\frac{R_{i j}^{\prime}}{R_{i j}}, k=1, \ldots, 119 .
$$

In each simulation experiment, we computed the means and the standard deviations of $r$. A total of 11,900 data points were produced from 100 reconstructions. A perfect reconstruction is represented by $r=1$. The ratios $r$ that were greater than or equal to 4.0 were treated as outliers. The means and standard deviations were computed without the outliers.

\section{Testing the Individual Stages of the Reconstruction}

Table 1 shows the means, standard deviations, and the numbers of outliers of $r$ for the shape hypotheses, cor-

\begin{tabular}{|c|c|c|c|}
\hline \multicolumn{4}{|c|}{$\begin{array}{l}\text { Means and Standard Deviations of the Ratios of Reconstructed } \\
\text { Distances and the Numbers of Outliers Obtained With Our } \\
\text { Model and With an Algorithm That Uses No Constraints }\end{array}$} \\
\hline \multirow[b]{2}{*}{ Reconstructed Shape } & \multicolumn{2}{|c|}{ Ratio } & \multirow[b]{2}{*}{ Outliers } \\
\hline & $M$ & $S D$ & \\
\hline Left-shape hypothesis & 1.097 & 0.304 & 55 \\
\hline Right-shape hypothesis & 1.101 & 0.302 & 57 \\
\hline Left corrected shape & 1.035 & 0.213 & 13 \\
\hline Right corrected shape & 1.042 & 0.246 & 31 \\
\hline Final shape reconstructed by the new model & 1.039 & 0.223 & 26 \\
\hline Reconstruction without constraints & 1.055 & 0.583 & 663 \\
\hline
\end{tabular}

Table 1

rected shapes, and final shapes obtained with our new model (which uses constraints) and for the shapes obtained with the algorithm of Chan et al. (1999). Chan et al.'s algorithm is the best that we identified from those that use visual data only (no constraints on the family of possible solutions). ${ }^{3}$ In this simulation experiment, vergence was $8^{\circ}$, and the noise standard deviation was $2 \%$.

It is seen that the new model, which uses constraints, is quite robust in the presence of noise. In fact, already the monocular shape reconstruction is substantially more stable than the binocular reconstruction obtained without constraints. Specifically, the standard deviations of the reconstructions representing the monocular shape hypotheses are about one half of the standard deviation of the binocular reconstructions without constraints. Moreover, the number of outliers in the case of monocular shape hypotheses is an order of magnitude smaller than the number of outliers obtained with the binocular reconstruction without constraints. These results suggest that in the presence of noise, constraints are more useful than binocular disparity. The correction involving the second image results in even more accurate reconstruction. This shows that our model can successfully use both the information in the images and constraints.

To verify whether our simulation experiments are likely to generalize to the case of shape reconstruction from real images, we built a wooden object whose shape was similar to those used in our psychophysical experiments (see Figure 7). Two images of the object were taken from viewing directions that were $8^{\circ}$ apart, and the object was reconstructed with our new model that uses constraints, as well as with Chan et al.'s (1999) algorithm, which does not use constraints. The coordinates of the vertices in the two images were measured by hand. It is seen that the reconstructed shape obtained with our new model is quite accurate and that the statistical parameters characterizing the accuracy of reconstruction are similar to those from the simulation experiments. The reconstruction without constraints is again much less accurate.

To verify whether that the results are likely to generalize to objects different from those that were used to formulate the model, we tested it with other objects as well. Figure 8 shows an example. Two views of a synthetic 

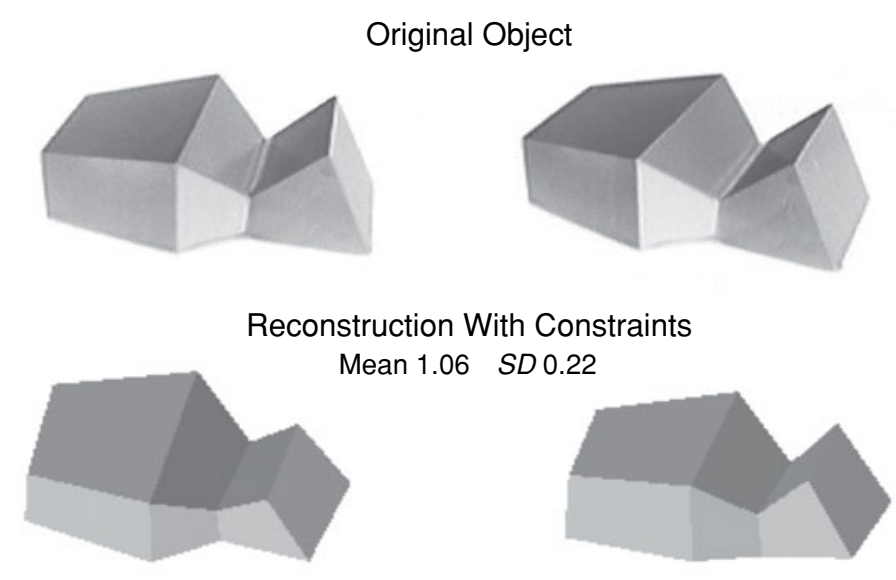

Reconstruction Without Constraints

Mean $0.55 S D 0.74$
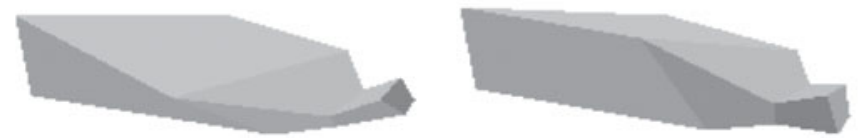

Figure 7. Real images of a wooden object and two different views of its reconstructions are shown. The means and the standard deviations of the normalized ratios are also shown.

object (a house) are shown on the top of Figure 8. We applied our model to two noisy images of this object. Simulated vergence was $8^{\circ}$, and noise standard deviation was $2 \%$ of eccentricity. Values of all parameters of the model were identical to those used in the experiments described above. It is seen that the shape reconstructed by the new model is similar to the original object. On the other hand, the shape reconstructed by the algorithm that does not use shape constraints is quite inaccurate.

\section{The Effect of Noise Standard Deviation}

The second set of simulation experiments tested the effect of visual noise. In this experiment, vergence was $8^{\circ}$, and the noise standard deviation was $1 \%, 3 \%$, and $5 \%$. Means, standard deviations, and the numbers of outliers for the final reconstruction obtained with our model and with an algorithm which uses no constraints are shown in Table 2.

It is seen that the binocular reconstruction with constraints (new model) is more stable and accurate than the binocular reconstruction without constraints for all three levels of noise. Specifically, at $1 \%$ of noise, which is similar to the amount of noise in the human visual system, our model produced only 1 outlier (ratio greater than 4.0). At the same time, binocular reconstruction without constraints produced 408 outliers. The average error and standard deviation of the reconstructions obtained with our model are $2 \%$ and $15 \%$, respectively, which are about four times better than those obtained with an algorithm that does not use constraints. It is worth pointing out that the variability of the reconstructions produced by the new model is very close to the variability of the reconstructions produced by human observers when they judged aspect ratios of generalized cylinders (Chan et al., 1999).

\section{The Effect of Viewing Distance}

The next simulation experiment tested the effect of the viewing distance. When the viewing distance is large the second image is not very useful, because the differences between the left and the right images produced by the differences in the viewing directions are small, in comparison with the distortions produced by noise. Table 3 shows results of reconstructions for three viewing distances corresponding to the vergence angle of $8^{\circ}, 4^{\circ}$, and $2^{\circ}$ (the results for $8^{\circ}$ vergence are taken from Table 1 ). It can be seen that the performance of our model is again more stable and that the numbers of outliers are substantially smaller than those obtained with an algorithm that uses no constraints.

\section{The Effect of the Object's Structure}

Finally, we tested the quality of the reconstructions for four types of objects that were used in our second psychophysical experiment. Recall from our psychophysical experiment that shape constancy, as measured by discriminability $d^{\prime}$, was most reliable for a polyhedron and least reliable for a nonplanar, asymmetric polyhedron (see Figure 4). We conjectured that the main reason for these differences in performance was the regularity of the shape itself. If a shape is regular, the visual system may apply stronger and more effective constraints, and this is likely to lead to more reliable reconstruction and shape constancy performance. An anonymous reviewer raised a question 


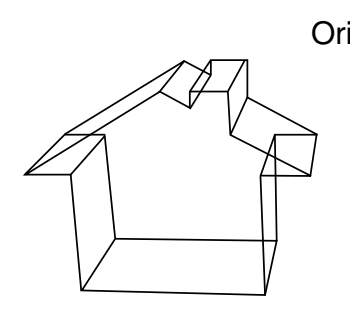

Original Object

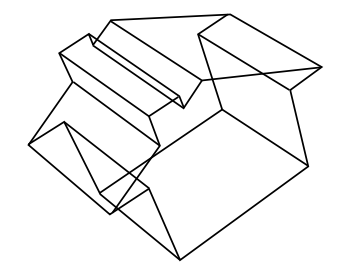

Reconstruction With Constraints Mean $0.92 S D 0.12$
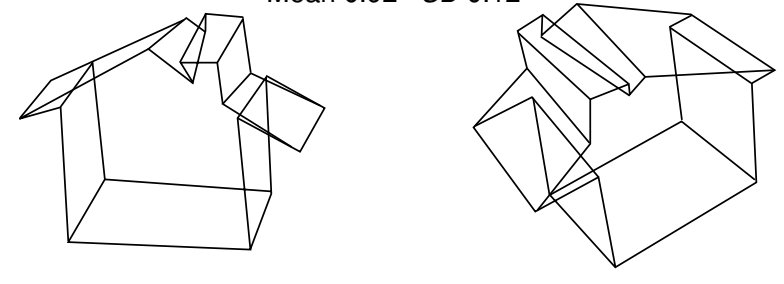

Reconstruction Without Constraints Mean $1.25 S D 0.22$
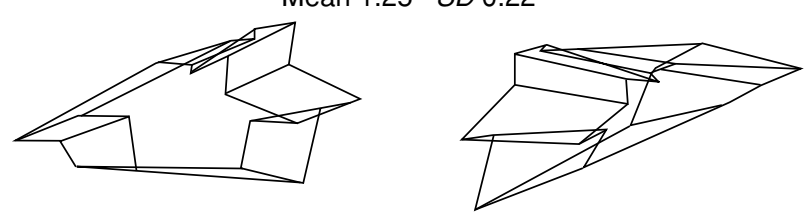

Figure 8. Two different views of the original object and its reconstructions are shown. The means and the standard deviations of the normalized ratios are also shown.

about how the visual system "knows" whether the shape is regular before the shape is reconstructed. Note that reconstruction of a 3-D shape in the visual system is likely to be preceded by analyzing the structure of a 2-D image. This is usually referred to as figure-ground organization. Depending on the content of the image, the visual system may decide which constraints, if any, to apply (Pizlo, 2001, pp. 3150-3151). For example, the presence of X junctions in the image provides strong evidence that the 3-D object is a polygonal line (see Figure 1C), and the presence of $\mathrm{Y}$ junctions in the image provides strong evidence that the 3-D object is a polyhedron (see Figure 1A).

If our computational model of binocular shape reconstruction were psychologically plausible, one would expect that the model's performance would be correlated with human performance. Note that we cannot (at this point) directly compare the performance of the model with that of the subjects because the model's output is a reconstructed shape, rather than a decision of same versus different. Considering that the stimuli in our psychophysical experiments shared the same overall 3-D Euclidean structure (16 vertices of a polyhedron), it is reasonable to expect that the variability of the ratios of reconstructed distances is inversely related to the proportion of correct responses.

Results from applying our new algorithm to the four shapes from the second psychophysical experiment are shown in Table 4. Simulated vergence was $8^{\circ}$, and noise standard deviation was $2 \%$. Table 4 shows the results for the left-shape hypothesis (the right-shape hypothesis produced very similar results) and for the final reconstruction. The results of the model without constraints (Chan et al., 1999) are not shown here simply because the performance of this model does not depend on the geometry of an object. It follows that its performance in the four conditions shown in Table 4 is equivalent to that shown in Table 1 (we verified this fact in simulations). It is seen that the stability of monocular reconstruction follows a pattern similar to that for $d^{\prime}$ in Figure 4. Specifically, stability of monocular reconstruction is best (i.e., the standard deviation of the ratio of reconstructed distances is lowest) in the case of a symmetric polyhedron that has planar faces and is substantially worse (by a factor of 2.5) in the case of asymmetric and nonplanar polyhedra. This suggests that the operation of constraints at the monocular stage provides a possible model for shape reconstruction in our shape constancy experiment. However, binocular correction leads to about equally good performance for all four types of stimuli. Specifically, binocular correction tends to improve stability of reconstruction for all stimuli (the correction was essentially absent in the case of the planar asymmetric shape), but the magnitude of the improvement is greater in the case of irregular shapes. This fact is interesting. It shows that constraints of minimum variance of angles, planarity, and mirror symmetry tend to improve binocular reconstruction not only in cases in which the object is symmetric and has planar contours, but also in cases in which objects are asymmetric and have nonplanar contours. In other words, the simplicity principle is effective not only in producing simple reconstructions, but also in producing veridical reconstructions.

The question arises as to why binocular shape constancy in our psychophysical experiment correlates better with monocular than with binocular shape reconstruction in our model. The most plausible explanation is that human shape constancy from novel views relies heavily on monocular shape reconstruction and the contribution of binocular disparity may be smaller than that produced by the current version of our model. The most direct way to check this possibility is to test subjects in shape constancy experiments as described above, but without a binocular disparity cue, and then to compare the performance produced when binocular disparity was absent with that

Table 2

Means and Standard Deviations of the Ratios of Reconstructed Distances and the Numbers of Outliers Obtained With Our Model and With an Algorithm That Uses No Constraints

\begin{tabular}{lccr}
\hline \multirow{2}{*}{ Noise Standard Deviation } & \multicolumn{2}{c}{ Ratio } & \\
\cline { 2 - 3 } & $M$ & $S D$ & Outliers \\
\hline New model (1\%) & 1.021 & 0.146 & 1 \\
Reconstruction without constraints (1\%) & 1.086 & 0.549 & 408 \\
New model (3\%) & 1.052 & 0.239 & 56 \\
Reconstruction without constraints (3\%) & 1.125 & 0.618 & 700 \\
New model (5\%) & 1.125 & 0.427 & 185 \\
Reconstruction without constraints (5\%) & 1.130 & 0.636 & 1289 \\
\hline
\end{tabular}

Note-Vergence was $8^{\circ}$, and noise standard deviation was $1 \%, 3 \%$, and $5 \%$. 
Table 3

Means and Standard Deviations of the Ratios of Reconstructed Distances and the Numbers of Outliers Obtained With Our Model and With an Algorithm That Uses No Constraints

\begin{tabular}{lcrr}
\hline \multirow{2}{*}{\multicolumn{1}{c}{ Vergence Angle }} & \multicolumn{2}{c}{ Ratio } & \\
\cline { 2 - 3 } & $M$ & $S D$ & Outliers \\
\hline New model $\left(8^{\circ}\right)$ & 1.039 & 0.223 & 26 \\
Reconstruction without constraints $\left(8^{\circ}\right)$ & 1.055 & 0.583 & 663 \\
New model $\left(4^{\circ}\right)$ & 1.115 & 0.362 & 117 \\
Reconstruction without constraints $\left(4^{\circ}\right)$ & 1.119 & 0.662 & 1008 \\
New model $\left(2^{\circ}\right)$ & 1.155 & 0.453 & 283 \\
Reconstruction without constraints $\left(2^{\circ}\right)$ & 1.203 & 0.710 & 1236 \\
\hline
\end{tabular}

Note-Vergence was $8^{\circ}, 4^{\circ}$, and $2^{\circ}$, and noise standard deviation was $2 \%$.

Table 4

Standard Deviations of the Ratios of Reconstructed Distances Produced by Our Model for the Stimuli in Experiment 2

\begin{tabular}{lcc}
\hline \multicolumn{1}{c}{ Stimulus Type } & $\begin{array}{c}\text { Left-Shape } \\
\text { Hypothesis }\end{array}$ & $\begin{array}{c}\text { Final } \\
\text { Reconstruction }\end{array}$ \\
\hline Polyhedron & 0.32 & 0.27 \\
Partially nonplanar, symmetric & 0.45 & 0.30 \\
Planar, asymmetric & 0.37 & 0.37 \\
Nonplanar, asymmetric & 0.79 & 0.26 \\
\hline Note-Vergence was $8^{\circ}$, and noise standard deviation was $2 \%$
\end{tabular}

Note-Vergence was $8^{\circ}$, and noise standard deviation was $2 \%$.

when binocular disparity was present. This is what we did in a control experiment described below.

We tested a naive subject and two of the authors, using the six types of stimuli from the first two experiments under two viewing conditions: binocular and monocular. Thus, the subjects ran a total of 12 sessions. To ensure that the viewing conditions were as similar as possible, the "monocular" sessions actually involved binocular viewing, except that all disparities were set to zero (the two stereoscopic images were identical; our informal observations showed that there was a very small, if any, difference between binocular viewing of two images having zero disparity and monocular viewing of a single image).
Since the absence of binocular disparity makes the figureground segregation problem more difficult when objects are transparent (see Figure 1A), we removed hidden edges in the case of the polyhedral stimuli. As was indicated by an anonymous reviewer, this modification can make a direct comparison between this control experiment and the three experiments described above difficult. Specifically, removing hidden edges may harm binocular performance because when the "back part" of the object is not visible, it cannot be used to perform shape discrimination. As a result, the pattern of binocular performance may change. This did not happen, however. Figure 9 shows the results for the naive subject (the results for the two authors were very similar). It can be seen that both the binocular and the monocular performances followed the pattern similar to that in our Experiments 1 and 2. Furthermore, although the binocular performance was always better than the monocular one, these two were strongly correlated. The correlation coefficient between monocular and binocular performance was quite high for the naive subject ( $r=$ .99). In the case of the other 2 subjects, Z.P. and Y.L., the correlations were .88 and .84 , respectively (see Pizlo, Li, $\&$ Chan, 2005, for more details). These high positive correlations between monocular and binocular performance suggest that binocular and monocular shape perception are based on a similar mechanism and that this mechanism likely involves shape constraints.

\section{SUMMARY}

Our psychophysical experiments generalized previous findings by showing that simplicity constraints are involved in shape constancy from novel views not only in the case of monocular viewing of a rotating object, but also in the case of binocular viewing of a stationary object. Constraints are involved in shape perception because the perception of 3-D shapes is a difficult inverse problem (Pizlo, 2001).

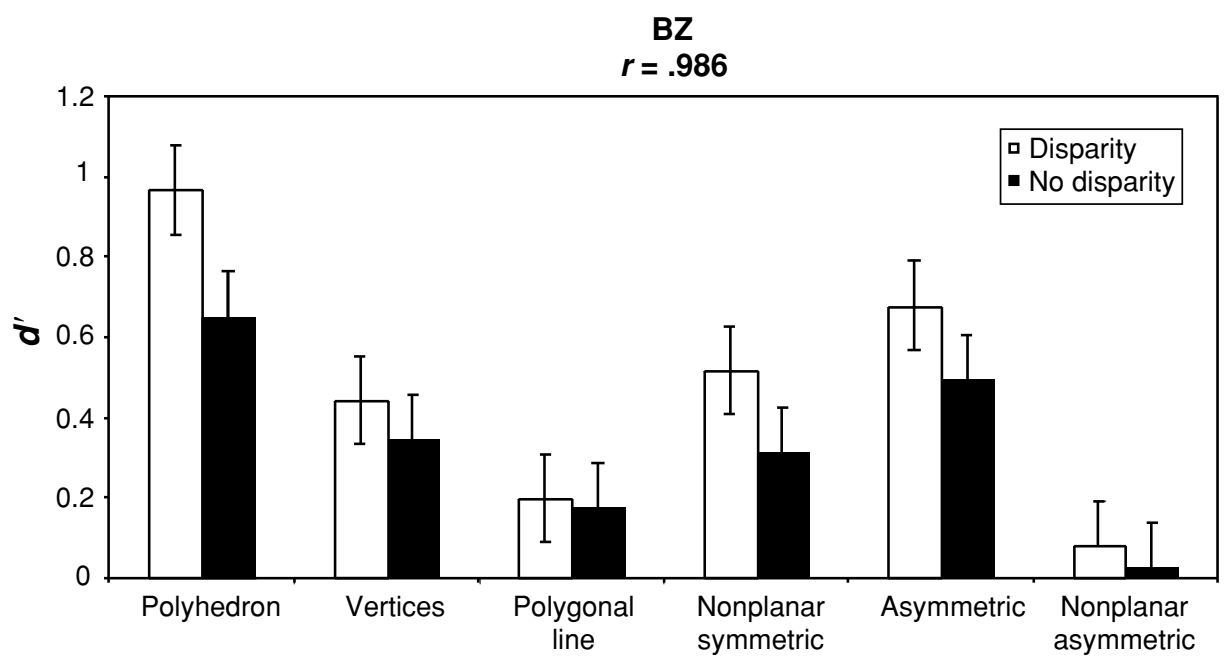

Figure 9: Results of the control experiment. 
To test the computational effectiveness of constraints in binocular shape reconstruction, we formulated a model and performed a set of simulations, using the same type of shapes as those used in our psychophysical experiments. In the model, binocular shape reconstruction begins with monocular reconstruction, and depth cues (binocular disparity) are used later on to correct the monocularly reconstructed shape. The performance of the new model was compared with the performance of one of the previous algorithms in which shape constraints were not used. This comparison showed that the new model is an order of magnitude more reliable. The reliability of the new model across four types of polyhedra was compared with the performance of human subjects. Monocular performance of the model correlated well with human performance; binocular did not. To shed more light on the relation between binocular and monocular shape constancy, a control experiment was performed, in which the subjects were tested under monocular and binocular viewing conditions. Performance in monocular viewing was strongly correlated with that in binocular viewing, suggesting that shape constraints are equally important in monocular and binocular vision.

The next step will be to modify the binocular correction stage of our existing model so that the reliability of binocular reconstruction in the model is correlated with human performance in the binocular shape constancy task. In order to directly compare performance of the model with that of human subjects, we will have to make one more elaboration of the model; namely, we will need to add a decision stage, so that the model, given two representations of 3-D shapes, will be able decide whether they are the same or different. This will allow comparing the $d^{\prime}$ for the model with that for the subjects.

\section{REFERENCES}

Anstis S. M. (1974). A chart demonstrating variations in acuity with retinal position. Vision Research, 14, 589-592.

Attneave F., \& Frost, R. (1969). The determination of perceived tridimensional orientation by minimum criteria. Perception \& Psychophysics, 6, 391-396.

BIEDERMAN, I. (1987). Recognition by components: A theory of human image understanding. Psychological Review, 94, 115-147.

Biederman, I., \& Gerhardstein, P. C. (1993). Recognizing depthrotated objects: Evidence and conditions for three-dimensional viewpoint invariance. Journal of Experimental Psychology: Human Perception \& Performance, 19, 1162-1182.

CHAN, M. W. (1999). A psychologically plausible algorithm for binocular shape reconstruction. Unpublished doctoral dissertation, Purdue University.

Chan, M. W., Pizlo, Z., \& Chelberg, D. M. (1999). Binocular shape reconstruction: Psychological plausibility of the 8-point algorithm. Computer Vision \& Image Understanding, 74, 121-137.

FAUGERAS, O. (1993). Three-dimensional computer vision: A geometric viewpoint. Cambridge, MA: MIT Press.

Hartley, R. I., \& Sturm, P. (1997). Triangulation. Computer Vision \& Image Understanding, 68, 146-157.

HochberG, J., \& Brooks, V. (1960). The psychophysics of form: Reversible perspective drawings of spatial objects. American Journal of Psychology, 73, 337-355.

Hochberg, J., \& McAlister, E. (1953). A quantitative approach to figural "goodness." Journal of Experimental Psychology, 46, 361-364.
Julesz, B. (1971). Foundations of cyclopean perception. Chicago: Chicago University Press.

Kersten, D., Mamassian, P., \& Yuille, A. (2004). Object perception as Bayesian inference. Annual Review of Psychology, 55, 271-304.

KoenderinK, J. J., van Doorn, A. J., \& KapPers, A. M. L. (1992). Surface perception in pictures. Perception \& Psychophysics, 52, 487-496.

LECLERC, Y. G. (1989). Constructing simple stable descriptions for image partitioning. International Journal of Computer Vision, 3, 73-102.

LECLERC, Y. G., \& FischleR, M. A. (1992). An optimization-based approach to the interpretation of single line drawings as 3-D wire frames. International Journal of Computer Vision, 9, 113-136.

LEVI, D. M., \& KLEIN, S. A. (1990). The role of separation and eccentricity in encoding position. Vision Research, 30, 557-585.

Liu, Z., \& Kersten, D. (2003). Three-dimensional symmetric shapes are discriminated more efficiently than asymmetric ones. Journal of the Optical Society of America A, 20, 1331-1340.

LongUeT-HigGins, H. C. (1981). A computer algorithm for reconstructing a scene from two projections. Nature, 293, 133-135.

Macmillan, N. A., \& Creelman, C. D. (2005). Detection theory: A user's guide (2nd ed.). Mahwah, NJ: Erlbaum.

MARILL, T. (1991). Emulating the human interpretation of line-drawings as three-dimensional objects. International Journal of Computer Vision, 6, 147-161.

McKee, S. P., Levi, D. M., \& Bowne, S. F. (1990). The imprecision of stereopsis. Vision Research, 30, 1763-1779.

Mitchison, G. (1988). Planarity and segmentation in stereoscopic matching. Perception, 17, 753-782.

Norman, J. F., Todd, J. T., Perotti, V. J., \& Tittle, J. S. (1996). The visual perception of three-dimensional length. Journal of Experimental Psychology: Human Perception \& Performance, 22, 173-186.

PerKINs, D. N. (1972). Visual discrimination between rectangular and nonrectangular parallelopipeds. Perception \& Psychophysics, 12, 396-400.

Perkins, D. N. (1982). The perceiver as organizer and geometer. In J. Beck (Ed.), Organization and representation in perception (pp. 7393). Hillsdale, NJ: Erlbaum.

Pizlo, Z. (2001). Perception viewed as an inverse problem: A minireview. Vision Research, 41, 3145-3161.

Pizlo, Z., LI, Y., \& Chan, M. (2005). Regularization model of human binocular vision. In Proceedings of IS\&T/SPIE Conference on Computational Imaging (Vol. 5674, pp. 229-240). Bellingham, WA: International Society for Optical Engineering.

Pizlo, Z., Li, Y., \& Francis, G. (2005). A new look at binocular stereopsis. Vision Research, 45, 2244-2255.

Pizlo, Z., Rosenfeld, A., \& Epelboim, J. (1995). An exponential pyramid model of the time-course of size processing. Vision Research, 35, 1089-1107.

Pizlo, Z., Rosenfeld, A., \& Weiss, I. (1995). Interdisciplinary study of visual invariants. In D. Dori \& A. Bruckstein (Eds.), Shape, structure and pattern recognition (pp. 118-127). Singapore: World Scientific.

Pizlo, Z., Rosenfeld, A., \& Weiss, I. (1997). The geometry of visual space: About the incompatibility between science and mathematics. Dialogue. Computer Vision \& Image Understanding, 65, 425-433.

Pizlo, Z., \& Stevenson, A. K. (1999). Shape constancy from novel views. Perception \& Psychophysics, 61, 1299-1307.

Poggio, T., Torre, V., \& Koch, C. (1985). Computational vision and regularization theory. Nature, 317, 314-319.

Pomerantz, J. R., \& Kubovy, M. (1986). Theoretical approaches to perceptual organization. In K. R. Boff, L. Kaufman, \& J. P. Thomas (Eds.), Handbook of perception and human performance: Vol. 2. Cognitive processes and performance (pp. 36-1 to 36-46). New York: Wiley.

Rawlings, S. C., \& Shipley, T. (1969). Stereoscopic acuity and horizontal angular distance from fixation. Journal of the Optical Society of America, 59, 991-993.

REgAN, D. (2000). Human perception of objects. Sunderland, MA: Sinauer.

Rock, I., \& DiVITA, J. (1987). A case of viewer-centered object perception. Cognitive Psychology, 19, 280-293.

RoCK, I., WHEELER, D., \& TUdOR, L. (1989). Can we imagine how objects look from other viewpoints? Cognitive Psychology, 21, 185-210.

SHEPARD, R. N., \& COOPER, L. A. (1982). Mental images and their transformations. Cambridge, MA: MIT Press. 
Sinha, P., \& Adelson, E. H. (1992). Recovery of 3-D shape from 2-D wireframe drawings [Abstract]. Investigative Ophthalmology \& Visual Science, 33, 825.

Tuan, B. S., \& Legge, G. E. (1998). The viewpoint complexity of an object-recognition task. Vision Research, 38, 2335-2350.

Vetter, T., Poggio, T., \& BÜlthoff, H. H. (1994). The importance of symmetry and virtual views in three-dimensional object recognition. Current Biology, 4, 18-23.

WitKin, A., Terzopoulos, D., \& KaAs, M. (1987). Signal matching through scale space. International Journal of Computer Vision, 1, 133-144.

\section{NOTES}

1. It is worth pointing out that Biederman and Gerhardstein (1993) argued against mental rotation as the mechanism underlying shape constancy. In fact, Shepard and Cooper (1982, p. 119), who introduced and studied the concept of mental rotation, also claimed that mental rotation is not likely to be involved in shape recognition. Recall that the mental rotation mechanism is assumed to involve a gradual change in the 3-D orientation of an imagined object, so that the object goes through all intermediate orientations between the start and the end. In contrast, the ability to imagine how an object looks like from a new viewing direction does not have to involve gradual change in the orientation. Instead, it may rely on some invariant features.

2. In principle, one might also try to apply constraints after the object has been reconstructed from binocular disparities. This method would be consistent with the model described by Julesz (1971), who showed, by using random dot stereograms, that the human visual system can produce a 3-D percept in the absence of monocular cues to depth. Note, however, that binocular shape reconstruction without constraints tends to be very noisy, especially when the viewing distance is of the order of several meters or more (see viper.psych.purdue.edu/binshape for examples illustrating the unreliability of shape reconstruction without constraints). It is more likely that the visual system performs multiple iterations, in which binocular disparity and monocular cues/constraints are applied recursively. As a result, if binocular disparity is not very effective because of a large viewing distance, monocular 3-D reconstruction may actually be performed first.

3. We also tried (1) Longuet-Higgins's (1981) algorithm, (2) its modification, in which the second part, where the actual reconstruction is performed, involved a triangulation method (Hartley \& Sturm, 1997), and finally, (3) the method commonly used by students of human vision, where the absolute depth of any point is computed as the sum of the absolute depth $D$ of the reference point and the relative distance (depth) $d$ between these two points. The relative depth $d$ is approximated on the basis of a binocular disparity of $\delta$ and an interocular distance of $I$ as follows:

$$
d \approx \frac{\delta \cdot D^{2}}{I}
$$

(Manuscript received May 28, 2004; revision accepted for publication October 25, 2005.) 